\title{
DEVELOPMENT OF A 3D WEBGIS SYSTEM FOR RETRIEVING AND VISUALIZING CITYGML DATA BASED ON THEIR GEOMETRIC AND SEMANTIC CHARACTERISTICS BY USING FREE AND OPEN SOURCE TECHNOLOGY
}

\author{
I. Pispidikis *, E. Dimopoulou \\ School of Rural and Surveying Engineering, National Technical University of Athens, 9 Iroon Polytechneiou str, 15780 Zografou, \\ Greece (pispidikisj@yahoo.gr; efi@ survey.ntua.gr)
} KEY WORDS: 3D WebGIS, CityGML, 3DcityDB, PostGIS, GeoServer, PHP, JQuery, AJAX, JavaScript, HTML5, WebGL, O13-
Cesium

\begin{abstract}
:
CityGML is considered as an optimal standard for representing 3D city models. However, international experience has shown that visualization of the latter is quite difficult to be implemented on the web, due to the large size of data and the complexity of CityGML. As a result, in the context of this paper, a 3D WebGIS application is developed in order to successfully retrieve and visualize CityGML data in accordance with their respective geometric and semantic characteristics. Furthermore, the available web technologies and the architecture of WebGIS systems are investigated, as provided by international experience, in order to be utilized in the most appropriate way for the purposes of this paper. Specifically, a PostgreSQL/ PostGIS Database is used, in compliance with the 3DCityDB schema. At Server tier, Apache HTTP Server and GeoServer are utilized, while a Server Side programming language PHP is used. At Client tier, which implemented the interface of the application, the following technologies were used: JQuery, AJAX, JavaScript, HTML5, WebGL and Ol3-Cesium. Finally, it is worth mentioning that the application's primary objectives are a user-friendly interface and a fully open source development.
\end{abstract}

\section{INTRODUCTION}

The rapid development of computer technology and the web have radically changed the data visualization techniques, hence leading to an increasing shift of improved 3D web visualization. Certainly, the advantages of $3 \mathrm{D}$ visualization did not leave the field of GIS unaffected, namely the implementation of 3D applications on $3 \mathrm{D}$ city models. It should be noted that through them, the spatial properties of urban objects can be understood in a more meaningful manner, facilitating the building of thinking, understanding and knowledge about the natural and human environment in geographic measurement scales (MacEachren \& Kraak, 2001). Worth noting the particular interest of the world community to the semantic and geometric modeling of 3D cities, which led to the adoption of an international standard, CityGML (Groger, Kolbe, Nagel, \& Hafele, 2012). Although it is considered as the most appropriate model for the representation of 3D city models, it is not efficient to present or visualize 3D city models directly on the web (Prandi, et al., 2015; Kanishk et al., 2015). Nevertheless, taking into account the benefits that the development of 3D WebGIS applications can generate by utilizing this standard, has led to the realization of several research investigations.

The objective of this paper is the development of a 3D WebGIS application in order to successfully retrieve and visualize CityGML data in accordance with their respective geometric and semantic characteristics in all Level of Details (LoDs).

To this purpose, a suitable class utilizing the Server-Side programming language PHP was initially developed. By this class the data received from PostGIS is achieved, based on both semantic characteristics and LoD of CityGML data. Hence, a
PostGIS Database is used, in compliance with the 3DcityDB schema, which supports multiscale and rich semantic structure of CityGML (Kolbe, et al., 2013). Next, a connection between GeoServer and PostGIS Database was established so as the portrayal of data is achieved through Open Geospatial Consortium (OGC) Web services, such as Web Map Service (WMS) and Web Feature Service (WFS). Finally, a web based client was developed, in order to support 2D/3D visualization and data management by utilizing open source technologies.

This paper is structured as follows. Section 2 briefly describes the available Web technologies. In section 3 related research projects are presented. Section 4 describes the methodology used in both Client and Server tier for the application to be implemented. Section 5 presents the abilities of WebGIS application. Finally, Section 6 concludes the whole paper and discusses suggestions for future research work.

\section{TECHNOLOGIES}

\subsection{D Web Technologies}

In previous years, the visualization of $3 \mathrm{D}$ graphics on the web was implemented through Application Programming Interfaces (API), which were supported only by specific web browsers or by using the appropriate plug-ins. The overcoming of the said limitation brought about the development of WebGL and Hyper Text Markup Language 5 (HTML5) technologies. In particular, the implementation of this approach enables $3 \mathrm{D}$ visualization in most popular web browsers, without the need of additional plugins to be installed (Iglesias, 2012).

\footnotetext{
* Corresponding author
} 
HTML5 is the fifth version of the HTML descriptive language. Moreover, new elements were added such as semantic, graphic and multimedia. In addition, the current version includes new API such as HTML Geolocation, HTML Drag and Drop, HTML Local Storage, HTML Application Cache, HTML Web Workers and HTML SSE (W3C HTML Working Group, 2014). More specifically, Canvas and Scalable Vector Graphics (SVG) are utilized for visualization propose, providing developers with tools for image and vector processing. Furthermore, via HTML Geolocation the real time spatial searches are enabled utilizing the user's location. Additionally, through APIS HTML Web Workers and HTML SET the performance of web applications is improved, reducing the waiting time (Taraldsvik, 2011).

WebGL is a Canvas extension of HTML5 and is used to develop web applications that require $3 \mathrm{D}$ visualization. Noted that it is API with regard to the $3 \mathrm{D}$ graphics, which was written in a lowlevel language and is based on the OpenGL ES 2.0 (Martin, 2011). The advantages of the use of WebGL is that it provides accelerated 3D functionality on the web, utilizing the Client graphics card, leading to a significant performance improvement (Taraldsvik, 2011).

HTML5 and WebGL technologies were widely used by researchers in order to solve complex problems. Regarding the implementation of 3D WebGIS systems with worldwide range, many virtual globes were developed, based on the above technologies such as Cesium, WebGL and Open Web Globe. These virtual globes are used as a tool for $3 \mathrm{D}$ visualization and interaction with global geospatial data, providing access to georeferenced satellite imagery (Kanishk, 2014). Additionally, they allow users to interact and retrieve data worldwide in real time (Elvidge \& Tuttle, 2008).

\subsection{Geospatial Web Services}

In 1993, the Xerox Corporation Palo Alto Research Center (PARC) developed a Web-Based map viewer, making the origin of WebGIS (Fu \& Sun, 2010). However, the technologies in which the WebGIS were implemented, have limitations both in their internal architecture as well as their integration with other information systems. As a result, the WebGIS were isolated (Huang, 2002). The solution to this isolation was brought about by web services, which constitute the heart of GIS and are the main reason for the progress of distributed GIS.

Service-Oriented Architecture (SOA) was implemented via the adoption of Web Services. Web service can be easier deployed and integrated in a distributed network environment. Certainly, the completion of the actual objectives of SOA came to be achieved by Web-Oriented Architecture (WOA), which is a specialization of SOA, utilizing RESTful Web services and lightweight mashups (Thies \& Vossen, 2008). Using WOA a full utilization of Web capacity was achieved and hence, the development of reliable, flexible application was facilitated in a most easiest and economical way

(Kralidis, 2007).

The OGC is an international non-profit organization committed to making quality open standard for the global geospatial community. The most significant geospatial Web services created by the said consortium are the Web Map Service (WMS), Web Feature Service (WFS), Web Coverage Service (WCS) and Catalogue Service for the Web (CSW). However, in the content of 3D except the WFS, there is no available service that has been standardized yet. Nevertheless, they are to be standardized the following 3D portrayal services: Web 3D Service (W3DS) (Quadt \& Kolbe 2005; Schilling \& Kolbe 2010) and Web View Service (WVS) (Benjarmin, 2010).

\section{RELATED RESEARCH WORK}

Considering the benefits that can be generated by the use of CityGML standard, there have been several research projects on the visualization of $3 \mathrm{D}$ city models.

Mao (2011) developed a framework for the visualization of 3D city models. As data source the CityGML was used, which turned into an Extensible 3D (X3D) scene and finally it was visualized on the web utilizing the pronounced X-Freedom (X3DOM). Specifically, CityGML data were analyzed and converted to Java Classes, representing various city objects such as buildings, streets, etc. The said conversion was implemented by the use of Citygml4j API. Then, the respective scenes were generated in accordance with geometric or semantic information.

Extending to the above architecture is the framework developed by Prieto et al., (2012). This research incorporates the usage of 3D web services such as W3DS instead of Java classes. Hence, a visualization of CityGML file was achieved without plugins. The output format was X3D and the integration into the web was achieved through X3DOM.

In 2012, several experiments were presented by 3D Portrayal Interoperability Experiment (3DPIE). These experiments were carried out utilizing the 3D portrayal services W3DS and WVS, which are on the way to be standardized (Schilling, Hagedorn, \& Coors , 2012)

In the context of the above experiments, the LSIS (Laboratoire des sciences de l' information et des systemes) laboratory focused on the representation of CityGML buildings and carried out three tests. In the first test, the entire CityGML file was fetched from a WFS server onHTML thick client based on C++. In the second test, the CityGML file was first processed on a server using Java Architecture for XML Binding (JAXB) parser. As a result, only the required part can be fetched on the client. In the last test, the CityGML stream was replaced with a JavaScript Object Notation (JSON) stream. This choice was made taking into account that the latter can be more easily portrayed on the web using Three.js API, which utilizes the WebGL technology (Schilling, Hagedorn, \& Coors, 2012).

Extending to the last test is the approach of Gesquiere \& Manin (2012), who adopted the tiled-based approach to work on CityGML files. The CityGML file was broken into several tiles and each tile was transformed into JSON, which was stored on the server. Hence, the client made requests to the server on the basis of specific tile and consequently, the server responded with JSON file for that file. Noted that the major advantage of the tilebased approach is that allows progressive visualization, which means, only the area required to be visualized will be fetched from the server (Kanishk, 2014).

Similarly, Prandi et al. (2013), following the tile-based approach, developed a framework in the context of a project called iSCOPE (interoperable Smart City Services through an Open Platform for urban Ecosystems). Specifically, they separated the CityGML files into tiles, storing them to the Server and finally, the Client can make requests based on the said requests. As a result, the progressive visualization was achieved. 
Additonally, Prandi et al. (2015) involved with the 3D web visualization of huge CityGML models, which were originally stored in the DB in compliance with 3DcityDB schema. Thereafter, in order the visualization of their data to be achieved and, in addition, their thematic features to be able to be searched, the following procedures were implemented: firstly, for visualization purpose the data was exported to Keyhole Markup Language/ COLLAborative Design Activity (KML/COLLADA) format together with the specific CityGML ID of the feature; secondly, the data was retrieved from the 3DcityDB utilizing the OGC WFS server using the CItyGML ID as query attributes.

Finally, CHATURVEDI et al. (2015) presented a Web based 3D client, which has been developed on top of WebGL based Cesium virtual globe utilizing the following technologies: ExtJS JavaScript-based web framework and HTML5. It should be noted that the highlighted features of the said client are the data exploration, the managing interaction and the queries based on the attributes of the data. The visualization of the data was achieved using KML/COLLADA files and JSON encoded data.

\section{METHODOLOGY}

The WebGIS application was developed in accordance with open source technologies. Moreover, based on the international trends and surveys, the CityGML was chosen as data source due to the fact that it is considered the most suitable standard for 3D city model visualization. In addition, PostgresSQL/PostGIS DB was used. At Server tier, Apache HTTP server and Geoserver were utilized, while server-side programming language PHP was chosen. At Client tier, the following technologies were used: JQuery, AJAX, JavaScript, HTML5, WebGL and O13-Cesium. Figure 1 shows the above technologies.

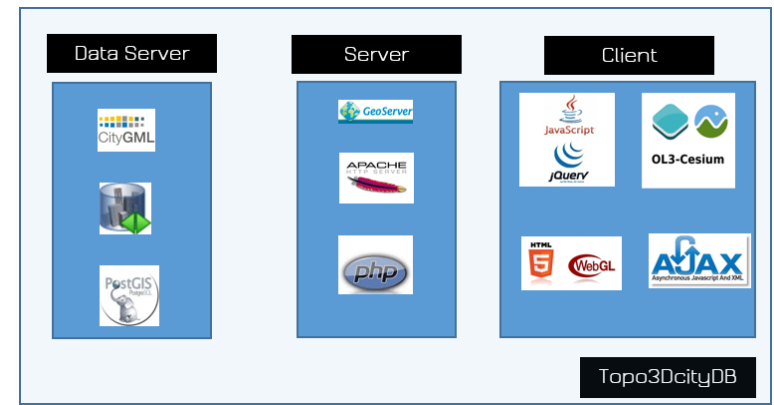

Figure 1. Server-side and Client-side technologies

\subsection{Pre-processing}

The 3DcityDB schema was originally installed, which supports the multi-scale and rich semantic structure of CityGML (Kolbe et al., 2013). Thereafter, CityGML data was chosen to be utilized, regarding buildings in LoD2 to LoD4. In addition, storing data into DB was achieved by the use of 3DcityDB importer/exporter (Kolbe et al., 2013). It should be noted that a connection between the DB and Geoserver was established in order that the portrayal of data is achieved through OGC Web services such as WMS and WFS. The pre-processing is schematically shown in Figure 2.

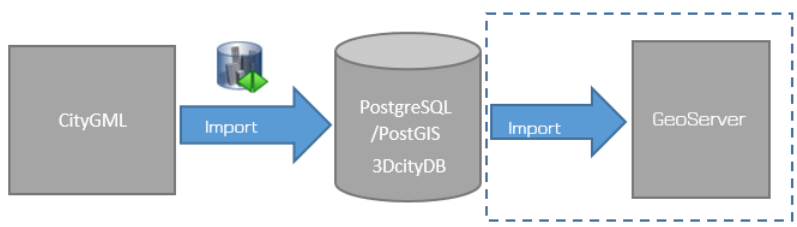

Figure 2. Pre-processing

\subsection{Client-Side Implementation}

The development of web based client is based on the Client-Side programming language JavaScript. The aforementioned technology is enriched by JQuery framework. In addition, the web based client is implemented utilizing the descriptive language HTML5. Additionally, as a means of representing 3D environments, the Cesium virtual globe is utilized. The Cesium virtual globe takes advantage of WebGL and HTML5 technologies plus it is considered suitable for visualizing data in a worldwide scale. In order to fully utilize the mapping capabilities of the OpenLayers API, the recently developed Ol3Cesium JS API is implemented (Ol3-Cesium, 2015). The Clientside architecture of the interface is shown in Figure 3.

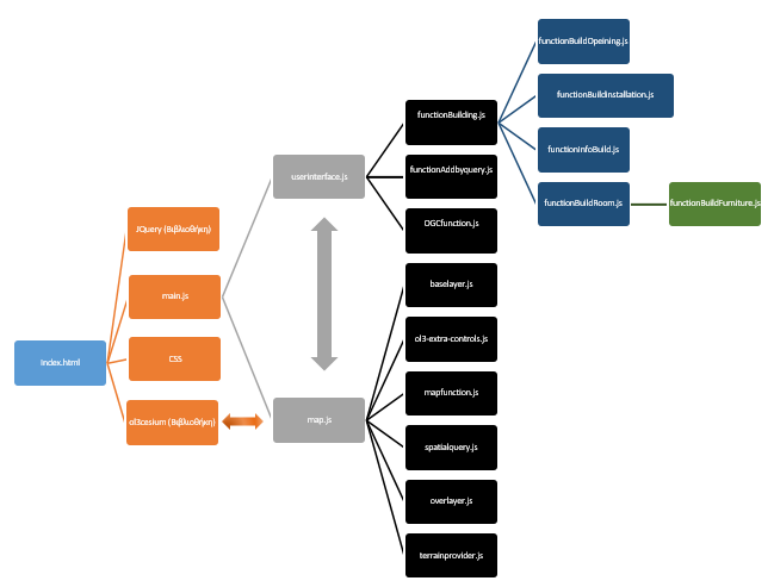

Figure 3: Client-side architecture of the interface

\subsection{Server-Side Implementation}

The retrieval and visualization of the data was achieved by utilizing two approaches. The first, uses the cityDBWrapper class, while the second is through Geoserver. The Class cityDBWrapper was developed utilizing the Server-Side programming language PHP. It should be noted that the development of the said Class is needed so that the receiving data from PostGIS is achieved, based on both semantic characteristics and LoD of CityGML data. Additionally, the functions of the aforesaid class return data in Array or JSON format and hence, it can be easily converted through the available functions of PHP. Specifically, the structure of the aforementioned class is shown in Figure 4. The asynchronous communication between Client and DB utilizing AJAX technology is shown in Figure 5. 


\begin{tabular}{|l|}
\hline \multicolumn{1}{|c|}{ cityDBWrapper } \\
\hline Server: text \\
Port: number \\
Schema: text \\
Database: text \\
Username: text \\
Password: text
\end{tabular}

Figure 4. Structure of cityDBWrapper Class

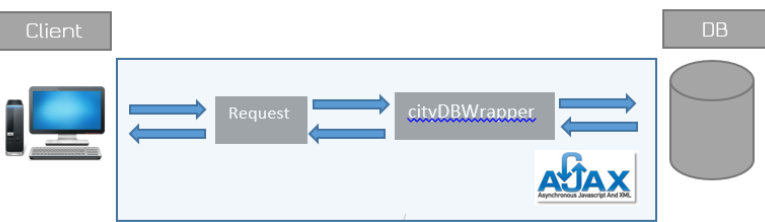

Figure 5. Asynchronous communication between Client and DB

Moreover, the Geoserver approach is implemented so as the visualization of data is achieved through OGC Web services. Therefore, a connection between the PostGIS DB and Geoserver was established. Thereafter, a suitable view was created by the use of SQL query shown in Figure 6. However, it is worth noting that via the Geoserver approach, data based on semantic characteristics has not been retrieved. Hence, the development of the class cityDBWrapper is necessary.

\section{SELECT}

ts.building_id,

ST_Collect(ST_Transform(sg.geometry,4326)) as geometry,

bd.usage,bd.usage_codespace,bd.roof_type,bd.measured_hei ght,bd.storeys_above_ground

\section{FROM}

surface_geometry sg,

thematic_surface ts,

building bd

WHERE ts.lod2_multi_surface_id=sg.root_id

AND ts.building_id=bd.id

AND ts.building_id is not NULL group by ts.building_id, bd.usage,bd.usage_codespace,bd.roof_type,bd.measured_hei ght,bd.storeys_above_ground order by ts.building_id

$$
\text { Figure 6. SQL query }
$$

\section{ABILITIES OF THE WEBGIS}

\subsection{Basic Application Abilities}

The WebGIS application was implemented by utilizing mashup technology. Consequently, many base maps and terrains can be used through the Web services (Figure 7). It is noteworthy that the choice of the terrains is enabled in 3D view.

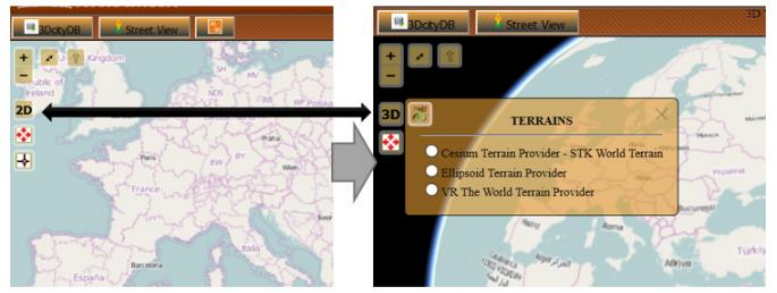

(a)

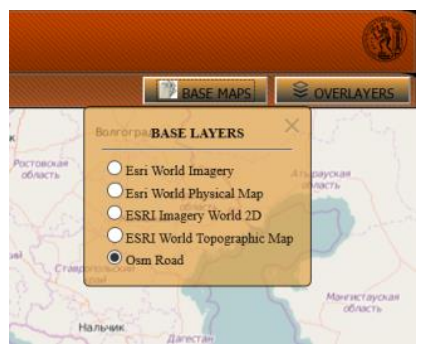

(b)

Figure 7. Terrain (a) and base maps (b) selection

5.1.1 Connection with 3DcityDB: The management and the visualization of the data are enabled, when a connection between the WebGIS application and the PostGIS DB is established. This connection is achieved filling out the respective form, which is provided by the WebGIS application (Figure 8). It should be noted that the PostGIS DB has to be used in compliance with 3DcityDb schema.

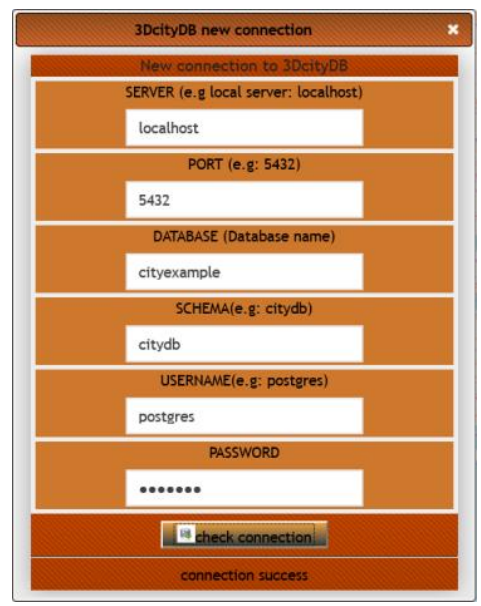

Figure 8. 3DcityDB connection form

5.1.2 Complex searches based on attribute data: Userfriendly combined queries utilizing attribute features are enabled to be executed. The users typically choose the LoD that suits their purpose. Thereafter, they are able to form the query based on attributed features without the need to know Structured Query Language (SQL) (Figure 9). 

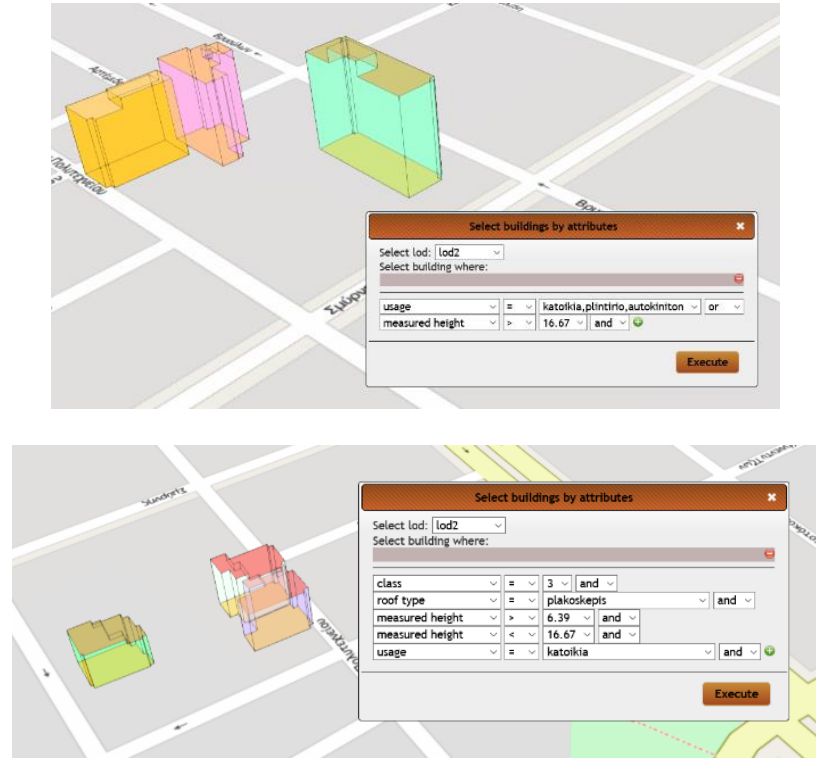

Figure 9. Combined non-spatial queries

5.1.3 Connection to Geoserver: A basic ability of the application is to retrieve and visualize data, which is served by Geoserver through the use of OGC Web services such as WMS and WFS. It should be noted that 3D portrayal services W3DS and WVS were not utilized since they have not been standardized yet and therefore, they are not supported by Geoserver. Furthermore, the JSONP (JSON with Padding) (Ippolito, 2005) was chosen as an output format from WFS. Thereafter, via an appropriate function, the retrieved geometries will be limited to the map extent. Consequently, the workload of the Client is significant reduced (Figure 10)

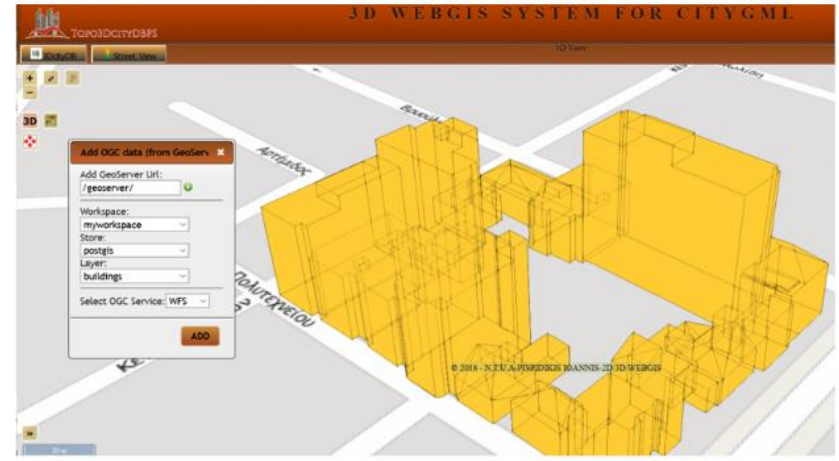

Figure 10. Retrieved data served by WFS

5.1.4 2D, 3D or 2D-3D view: The available data can be visualized in 2D, 3D or simultaneous 2D-3D view (Figure 11).

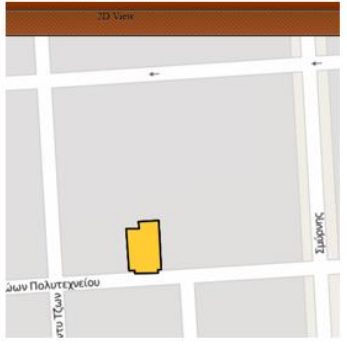

[a]

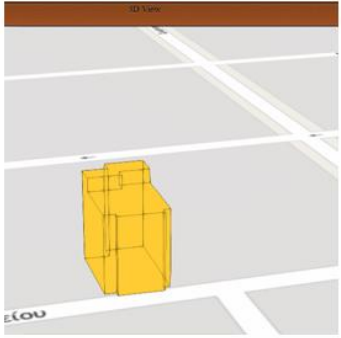

(b)

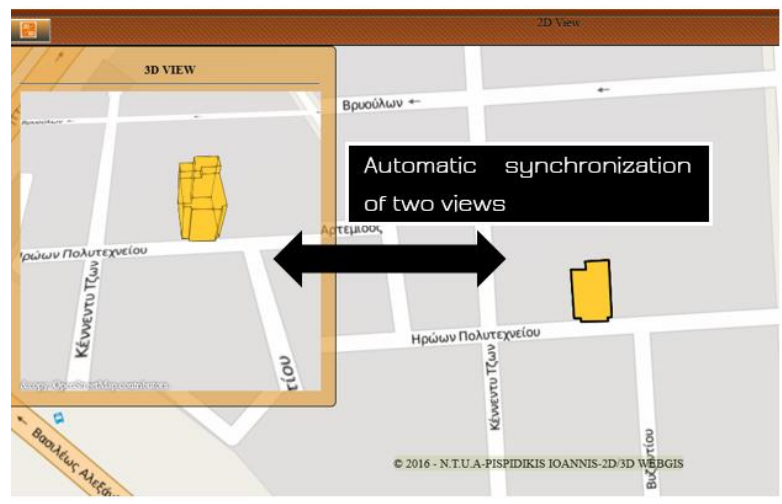

(c)

Figure 11. 2D (a), 3D (b) and 2D-3D view (c)

5.1.5 Spatial queries: The users are able to execute spatial queries in accordance with point and polygon geometries and based on the LoDs (Figure 12). Thus, if the data is retrieved successfully, a suitable list of available data will be displayed. Afterwards, the users can add this data to the application regarding the respective semantic characteristics.

5.1.6 Street View: This feature allows the users to view the study area through panoramic images of google maps. Simultaneously, the available semantic features of the respective buildings can be compared with the above panoramic images (Figure 13).

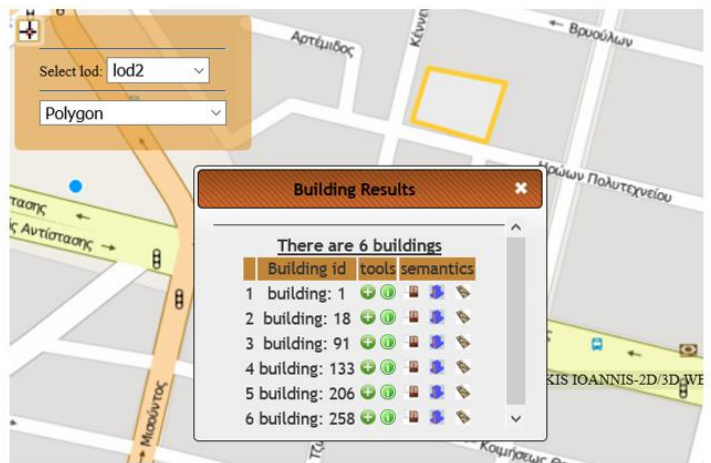

Figure 12. Spatial Queries 


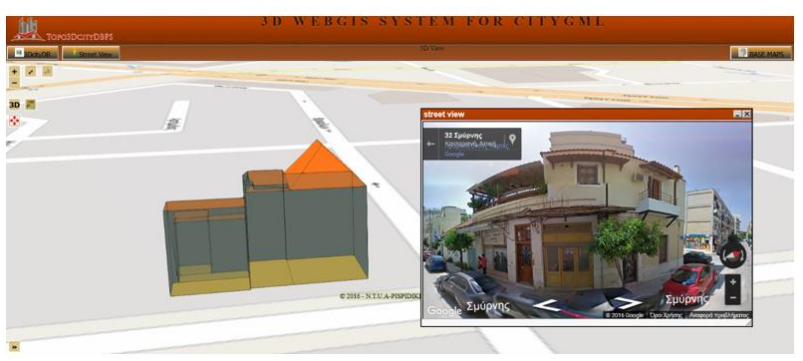

Figure 13. Street View

\subsection{Data Retrieval based on Semantic Characteristics}

The primary objective of this WebGIS application is the ability of the user to easily retrieve and visualize CityGML data in accordance with their respective geometric and semantic characteristics from 3DcityDB. Thereby, the users can style their semantic features (color and transparency) as well as being able to view the respective building, address and generic information (Figure 14). Moreover, they are able to retrieve data from many Databases, which have different reference system. Consequently, all data are visualized in a common reference system, WGS84. Examples of data in different LoDs are shown in Figure 15.

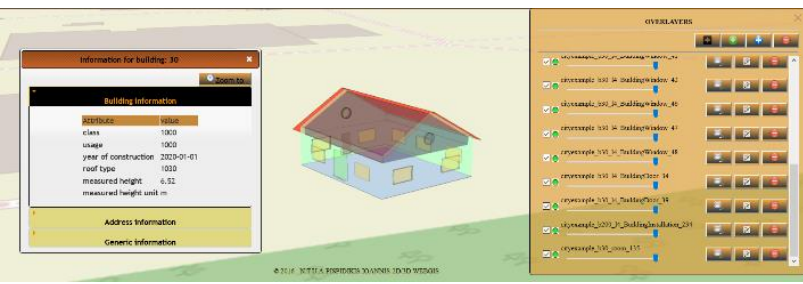

Figure 14. Building, address and generic information. Styling abilities
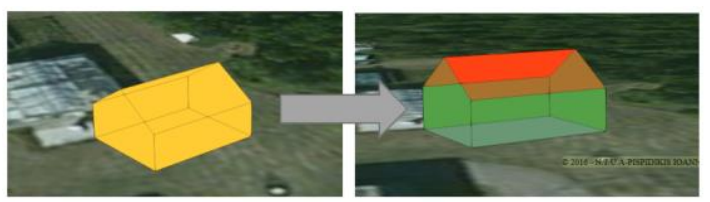

(a)
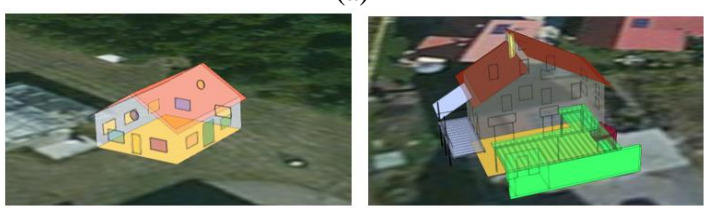

(b)

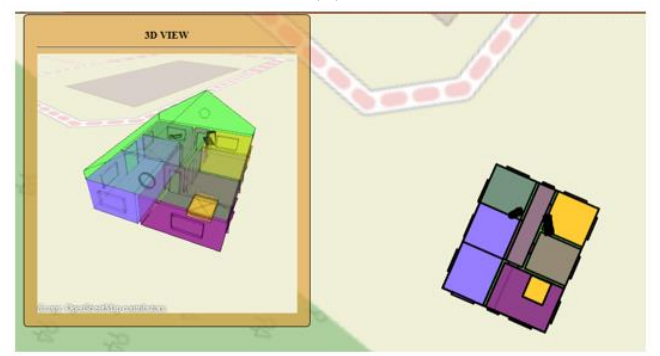

(c)

Figure 15. LoD 2 (a), LoD3 (b), LoD4 (c)

\section{CONCLUSION}

In the context of this paper, a 3D WebGIS application is developed in order to successfully retrieve and visualize CityGML data in accordance with their respective geometric and semantic characteristics. Initially, the CityGML model, which is considered as the optimal standard for representing 3D city models, is thoroughly studied. As results from the study, its format is not web friendly in terms of 3D data visualization and the paper proceeded to organize data in a PostGIS DB in compliance with the 3dCityDB schema. Next, the issue of data retrieval from the DB is addressed. Current research trends point to the fact that the optimal way to visualize 3D data in a WebGIS environment is through 3D portrayal services like W3DS and WVS. However, these services are not yet OGC standards and thus were not implemented since they are not supported from Web Servers such as GeoServer. Furthermore, the primary objective of this paper is not the visualization of large CityGML data but the retrieval and visualization of data based on specific semantic characteristics. In order to achieve the aforementioned goal, a server side scripting language was used, mainly PHP, to develop a class which utilizes AJAX techniques in order to dynamically retrieve data in JSON format.

Proposals for future research and development may include:

- Utilization of PHP class cityDBWrapper via suitable Web services.

- Extending of PHP class cityDBWrapper, so as to enable the retrieval of semantic features not only of the building model but also of the other CityGML models.

- Considering the benefits that can be brought about by modeling cities in different fields, the extension of the WFS needs to be examined. Specifically, additional functionalities could be added in regard to the retrieval of semantic data at any LoD. As a result, the retrieval, management and editing of data will be achieved via WFS and WFS-T.

- Further study of 3D portrayal services W3DS and WVS is also required since in the near future they will also become OGC standards.

\section{REFERENCES}

Benjarmin, H. (2010). Web View Service Discussion Paper. ver 0.3.0. OpenGIS Discussion Paper.

CHATURVEDI, K., YAO, Z., \& KOLBE, T. H. (2015). Webbased Exploration of and Interaction with Large and Deeply Structured Semantic 3D City Models using HTML5 and WebGL. In Wissenschaftlich-Technische Jahrestagung der DGPF und Workshop on Laser Scanning Applications (Vol. 3).

Elvidge, D. C., \& Tuttle, T. B. (2008). How virtual globes are revolutionizing earth observation data access and integration. The International Archives of the Photogrammetry, Remote Sensing and Spatial Information Sciences(37), pp. 137-139.

Fraunhofer. (2015). official x3dom documentation. Retrieved from x3dom (2016): http://doc.x3dom.org/

Fu, P., \& Sun, J. (2010). Web GIS: principles and applications. USA: Esri Press. 
Gesquiere, G., \& Manin, A. (2012). 3D Visualization of Urban Data Based on CityGML with WebGL. International Journal of 3-D Information Modeling (IJ3DIM), 1(3), pp. 1-15.

Groger, G., Kolbe, T., Nagel, C., \& Hafele, K.-H. (2012, March 4). OGC City Geography Markup Language (CItyGML) Encoding Standard. Retrieved from Open Geospatial Consortium Inc (2016): www.opengis.net/spec/citygm1/2.0

Huang, X. (2002). GeoAjent-based geospatial information service and application integration. Beijing: Phd diss.,Beijing University.

Iglesias, D. (2012). Design and Implementation of $3 D$ buildings integration for a WebGL-Based virtual Globe

. Retrieved from run (2016): http://hdl.handle.net/10362/8327

Ippolito , B. (2005). Remote JSON - JSONP. Retrieved from Bob Ippolito (2016): http://bob.ippoli.to/archives/2005/12/05/remotejson-jsonp/

Kanishk, C. (2014). Web based 3D analysis and visualization using HTML5 and WebGL. Enschede: Geo-information Science and Earth Observation (ITC), University of Twente.

Kolbe, T. H., Konig, G., Claus, N., \& Stadler, A. (2013). 3D City Database for CityGML Version 2.1.0. Institute for Geodesy and Geoinformation Science, Technische Universität Berlin. Retrieved from 3DcityDb (2016): http://www.3dcitydb.net/3dcitydb/fileadmin/downloaddata/3DC ityDBDocumentation-Addendum-v2_1.pdf

Kralidis, T. A. (2007). Geospatial web services: the evolution of geospatial data infrastructure. Springer London.

MacEachren, M. A., \& Kraak, J. M. (2001). Research Challenges in Geovisualization. Cartography and Geographic Information Science, 28(1), pp. 3-12.

Mao, B., \& Ban, Y. (2011). Online Visualisation of a 3D City Model Using CityGML and X3DOM. Cartographica, Vol. 46, No. 2, pp. 109-114.

Martin, C. (2011). WebGL Specification, Khronos WebGL Working Group. Retrieved from Khronos (2016): http://www. khronos. org/webgl

Ol3-Cesium. (2015). Ol3-Cesium. Retrieved from OpenLayers (2016): http://openlayers.org/ol3-cesium/

Prandi, F., De Amicis, R., Piffer, S., Soave, M., Cadzow, S., Gonzalez Boix, E., \& D'Hont, E. (2013). Using CityGML to deploy Smart-City services for Urban Ecosystems. International Archives of the Photogrammetry, Remote Sensing and Spatial Information Sciences, Volume XL-4/W1, pp. 87-92.

Prandi, F., Devigili, F., Soave, M., Di Staso, U., \& De Amicis, R. (2015). 3D web visualization of huge CityGML models. ISPRSInternational Archives of the Photogrammetry, Remote Sensing and Spatial Information Sciences, 1, pp. 601-605.

Prieto, I., Izkara, L. J., \& del Hoyo, F. (2012). Efficient visualization of the geometric information of CityGML: application for the documentation of built heritage. Computational Science and Its Applications-ICCSA 2012, pp. 529-544.
Quadt, U., \& Kolbe, T. (2005, Febrouary 2). Web 3D Service.

Schilling, A., \& Kolbe, T. H. (2010). Draft for Candidate OpenGIS Web 3D Service Interface Standard. ver. 0.4.0. OpenGIS Discussion Paper.

Schilling, A., Hagedorn, B., \& Coors, V. (2012, August 27). OGC 3D Portrayal Interoperability Experiment FINAL REPORT. OGC Engineering Report.

Taraldsvik, M. (2011). Exploring the Future: is HTML5 the solution for GIS Applications on the World Wide Web? technical report, NTNU. Retrieved from http://mats.taraldsvik.net/gd12/publications/html5andgis.pdf (2016)

Thies, G., \& Vossen, G. (2008). Web-oriented architectures: On the impact of Web 2.0 on service-oriented architectures. IEEE Asian-Pacific Services Computing Conference, (pp. 1075-82).

W3C HTML Working Group. (2014, October 28). HTML5. A vocabulary and associated APIs for HTML and XHTML. Retrieved from W3C (2016): http://www.w3.org/TR/html/ 